\title{
Pragmaticalisation parallèle des marqueurs discursifs : le cas de déjà
}

\author{
Gerda Haßler \\ Université de Potsdam, Allemagne \\ hassler@uni-potsdam.de
}

Résumé. Les marqueurs discursifs ont subi un processus de pragmaticalisation, un cas particulier de grammaticalisation, dans lequel ils ont été décatégorisés et ont acquis une fonction en dehors de la phrase. Cette fonction leur permet d'établir des relations entre des énoncés et de marquer la valeur informative de certaines parties d'un énoncé. Quand on observe la fonction des étyma des ces éléments en latin (classique et non-classique), on peut constater un usage des particules en question que l'on retrouve en français contemporain. Ainsi iam dans l'exemple suivant sert de connecteur et ne remplit aucune fonction dans la phrase : Iam id porro utrum libentes an inviti dabant? (Cic. Ver. 3.118.). Il y a des mots latins qui fonctionnaient déjà comme marqueurs discursifs et qui ont élargi leur fonction au cours de l'histoire. Est-ce que cette constatation remet en question leur étude en termes de grammaticalisation ou de pragmaticalisation? Nous voulons démontrer que cela n'est pas le cas. Chacune des particules étudiées connaît une période de 'réinvention' en tant que marqueur discursif, c'est-à-dire qu'on ne peut pas constater un usage continu à partir du latin. Ce fait est certainement dû au manque de textes oraux ou de textes qui ont un certain trait d'oralité simulée.

\begin{abstract}
Discourse markers emerge from a process of pragmaticalization. We treat pragmaticalization as a special case of grammaticalization, in which discourse markers are decategorized and change their meanings so that they can assume a function outside the sentence. This function allows them to create relations between utterances and to mark the informational value of certain parts of an utterance. When we examine the function of these elements' etyma in either classical or non-classical Latin, we can observe a use of these particles that occurs also in present-day French. In this way, iam serves as a connector in the following examples and has no function within the sentence: Iam id porro utrum libentes an inviti dabant? (Cic. Ver. 3.118.) In Latin there were already words which served as discourse markers and which have extended their function in the course of their development. Does this present a problem for considering their development as instances of grammaticalization and pragmaticalization? We seek to demonstrate that this is not the case. Each of the particles under consideration went through a period of "reinvention" as a discourse marker; that is, we cannot establish any continuous use beginning with Latin. This discontinuity is no doubt in part due to the lack of oral texts or texts that seek to imitate spoken language. The differentiation of the meanings of discourse markers would seem to be a panchronic phenomenon, which has a cognitive basis, but which must first become established in individual languages. In the Romance languages this development proceeded over the course of several centuries and began at different points in time. Despite this, the development occurred independently of mutual influences between the language but still led to largely the same result.
\end{abstract}




\section{Pragmaticalisation parallèle : explication du concept}

Dans cette contribution, je vais me concentrer sur l'analyse d'un marqueur discursif qui provient d'un adverbe temporel : ja avec sa forme nouvelle déjà. Dans notre définition d'un marqueur discursif, nous suivons Mosegaard Hansen (1998: 73) qui le définit comme «non-propositional linguistic item whose primary function is connective, and whose scope is variable». Buchi préfère le terme pragmatème qui désigne une unité pragmatique qui s'oppose tant au lexème qu'au grammème "par la particularité que son rôle se situe non pas sur le plan reférentiel, mais sur le plan communicatif » (Buchi 2007 : 352 ; cf. Dostie 2004 : 27). Pour le processus du développement des marqueurs discursifs, nous utilisons le terme pragmaticalisation, calqué sur l'anglais pragmaticalization qu'on peut définir comme un processus par lequel une unité linguistique aquiert le statut d'une unité pragmatique (Buchi 2007 : 352)

On pourrait déduire de la lecture de certains travaux sur les marqueurs discursifs que la formation de ces éléments linguistiques est un processus récent qui se déroule à l'intérieur d'une langue, dans notre cas le français. Par ailleurs, on cherche souvent à déterminer, à partir de leur «signification de base », les directions dans lesquelles les significations des marqueurs discursifs ont évolué. Ainsi, par exemple Mosegaard Hansen \& Strudsholm (2008) considèrent que, si les données diachroniques montrent qu'une signification B apparaît nettement plus tard qu'une signification A, on peut les considérer comme des sens différents d'une forme polysémique. Si deux significations C et D, par contre, coexistent dans tous les stades de l'histoire d'une langue et que la différence interprétative peut être attribuée au contexte linguistique, il faut alors partir de l'hypothèse de modulations contextuelles du même sens :

[...] if our diachronic data show that use B appears substantially later than use A, we will assume as a working hypothesis that they are distinct senses, whereas if two uses, $\mathrm{C}$ and $\mathrm{D}$, appear to coexist at any historical stage, and the interpretative difference between them can be attributed to linguistic context, then we assume - again as a working hypothesis - that they are in fact different contextual modulations on one and the same sense. (Mosegaard Hansen \& Strudsholm 2008, 475)

Nous voulons montrer que la prise en compte de l'usage des marqueurs dans des textes latins et dans des textes des époques plus anciennes peut suggérer une troisième hypothèse. Deux significations $(\mathrm{E}$ et $\mathrm{F})$ peuvent être observables dès les premiers textes sans être provoquées par des contextes différents. Dans ce cas, nous parlons d'une grammaticalisation ou d'une pragmaticalisation parallèle qui consisterait en l'usage d'un adverbe dans sa signification temporelle et, parallèlement, en l'usage de la même forme comme marqueur discursif. ${ }^{1}$

Nous concevons la notion de pragmaticalisation parallèle d'une manière semblable à Nørgård-Sørensen, Heltoft \& Schøsler : «Connecting grammaticalisation - seen as a process of historical grammatical change - is about the development of such integrated and closed sets of paradigmatic systems. We distinguish two types of connecting grammaticalisation: complex and parallel paradigms $(2011,105)$ ».

\footnotetext{
${ }^{1}$ Sur la grammaticalisation des marqueurs discursifs cf. Combettes, Schnedecker et Theissen (2003), Dostie (2004), Marcello-Nizia, (2006), Waltereit (1998, 2006, 2012), Wouden, Foolen et Craen (2003).
} 
En parlant des marqueurs discursifs, nous préférons le terme de pragmaticalisation parce que l'affaiblissement du sens qu'ils subissent ne mène pas à des morphèmes grammaticaux, mais à un rôle discursif. Les marqueurs ont subi un processus de pragmaticalisation - un cas particulier de grammaticalisation, dans lequel ils ont été décatégorisés et ont acquis une fonction en dehors de la phrase. Cette fonction leur permet d'établir des relations entre des énoncés et de marquer la valeur informative de certaines parties d'un énoncé. Nous étudierons d'abord l'usage de la particule iam dans des textes latins, pour le comparer avec la pragmaticalisation de jàldéjà en français. Pour montrer le parallélisme panchronique des changements sémantiques au cours de la pragmaticalisation, nous partirons des significations trouvées dans les exemples latins et nous ne séparerons pas chronologiquement ces processus pour ja et déjà.

\section{La diversité des fonctions de iam en latin}

Dans son manuel de sémantique et syntaxe latines (Lehrbuch der lateinischen Syntax und Semantik), Hermann Menge cite déjà en 1873 des exemples qui ne sont pas restreints à la signification de iam comme adverbe temporel :

(1) Pons in Hibero prope effectus nuntiabatur; iam vero eo magis illi maturandum iter existimabant. (César, Civ. 1.62,3f. Menge 2000, 211)

'On avait annoncé que le pont à travers l'Ebro serait presque terminé; alors ils croyaient qu'ils devraient se dépêcher d'autant plus.'

Dans cet exemple, iam sert à la transition, il introduit une autre action et organise le discours. En même temps, la signification temporelle est toujours reconnaissable : on a tiré la conclusion qu'il fallait se dépêcher au moment où la construction du pont était sur le point d'être terminée. Il s'agit d'un de ces contextes qu'on peut dénommer par la métaphore métalinguistique contextes en pont et qui sont caractéristiques de la prise en charge d'une fonction discursive organisant le texte avec un adverbe temporel. De plus, déjà dans la langue latine, iam peut être utilisé d'une manière renforçante dans des phrases conditionnelles sans conjonction subordonnante :

(2) Recognosce mecum noctem illam superiorem: iam intelleges multo me vigilare acrius ad salutem quam te ad perniciem rei publicae (Cicéron, Catil. 1,8. Menge 2000, 832)

'Rappelle à ta mémoire l'avant-dernière nuit, et tu comprendras que je veille encore avec plus d'activité pour le salut de la république que toi pour sa perte.'

Ces deux propositions ne peuvent pas être liées par la conjonction et, mais elles se posent l'une après l'autre d'une manière asyndétique. Dans la première qui remplace une phrase conditionnelle, l'impératif est utilisé ; dans la deuxième le caractère coercitif de la compréhension est souligné par iam.

Dans des travaux traditionnels sur iam, très fréquents dans des oeuvres littéraires en latin, on trouve plusieurs significations qui sont attribuées à cette particule et qui divergent largement :

$1^{\circ}$ 'déjà depuis longtemps' (iam diu, iam pridem, iam dudum)

$2^{\circ}$ 'maintenant, tout de suite' (iam vero)

$3^{\circ}$ 'dans la suite'

$4^{\circ}$ 'même, en fait'

$5^{\circ}$ 'encore' (après une négation) (nec iam arma nec vires suppetunt « il n’y a ni assez d'armes, ni de forces suffisantes »)

$6^{\circ}$ dans des locutions : non iam 'ne pas plus', iam...iam 'tantôt...tantôt', iam olim 'depuis longtemps', vix iam 'presque plus', iamiam 'aussitôt'.

Il n'y a pas de discussions sur l'usage de iam comme adverbe temporel auquel d'autres expressions temporelles peuvent se joindre :

(3) $[\ldots]$ eius libertum Apollonium iam tum equidem, cum ille viveret, et magni faciebam et probabam [...] (Cic. Fam. 13.16.1. Kroon \& Risselda 2003, 66)

'même lorsqu'il [Crassus] vivait toujours, j'avais beaucoup de respect et de sympathie pour le libéré Apollonius' 
Kroon \& Risselda voient une différence minimale entre cet usage purement temporel et les exemples dans lesquels iam est une particule scalaire. Dans ces cas, le locuteur se réfère à l'échelle graduée de la durée et explique que le point atteint sur cette échelle est plus haut que ce qu'il attendait:

(4) sex menses iam hic nemo habitat (Plaut. Mast. 954. Kroon \& Risselda 2003, 66) 'déjà six mois que personne n'habite ici'

Mais l'échelle de référence n'est pas obligatoirement temporelle, comme le montre l'exemple suivant:

(5) non cum senatu modo sed iam cum dis immortalibus C. Flaminium bellum gerere (Livius 21.63.6. Kroon \& Risselda 2003, 66)

'que Gaius Flaminius était en guerre non seulement avec le Sénat, mais déjà avec les dieux immortels'

Les dieux se considèrent ici comme plus hauts dans l'échelle d'estime que les ennemis du Sénat. Cet usage scalaire et non restreint à la temporalité de la particule est donc un phénomène ancien qui n'a pas attendu pour apparaître la naissance des langues romanes. L'échelle de qualité sur laquelle un point de référence est dépassé peut être considérée comme contexte en pont pour cette signification.

De plus, Kroon \& Risselda $(2003,67)$ ont constaté que iam peut être utilisé comme connecteur. Ainsi, chez Cicéron on trouve la phrase interrogative suivante dans l'argumentation pour prouver un chantage :

(6) Iam id porro utrum libentes an inviti dabant? (Cic. Ver. 3.118. Kroon \& Risselda 2003, 67) 'Alors ils payaient ça consentants ou à contrecœur ?'

Iam ne remplit aucune fonction dans cette phrase et il ne modifie pas non plus la signification du verbe. Il structure plutôt le discours en liant la phrase avec l'argumentation antérieure.

Par rapport à la base théorique de notre contribution, cet exemple nous permet deux remarques :

$1^{\circ}$ Tandis que pour le sens d' « avoir dépassé un point sur une échelle temporelle ou non-temporelle », on peut expliquer le changement de la signification par des modulations contextuelles du même sens, dans l'usage comme connecteur, cela ne paraît pas possible. Il s'agit donc de deux significations $\mathrm{E}$ et $\mathrm{F}$ qui sont déjà présentes en latin et qui réapparaissent dans l'histoire du français jusqu'à aujourd'hui.

$2^{\circ}$ L'usage de iam comme connecteur apparaît surtout dans des textes qui sont marqués par l'oralité conceptuelle. En utilisant le terme oralité conceptuelle, nous suivons la théorie développée par Peter Koch et Wulf Oesterreicher (1990), consistant à introduire une distinction entre une oralité / scripturalité médiale et une oralité / scripturalité conceptuelle. Le côté du médium est structuré de façon binaire : un acte communicatif est produit ou bien dans le code graphique et donc médialement écrit, ou bien dans le code phonique et donc médialement oral. La conception de cette production peut s'échelonner entre deux pôles, entre une oralité conceptuelle et une scripturalité conceptuelle (cf. Pusch 2007). Il est donc fort probable que l'usage de iam comme connecteur et marqueur discursif était courant dans le latin parlé dont nous pouvons observer des traces dans quelques documents écrits non officiels ou même dans des œuvres de grands écrivains qui l'utilisent pour simuler l'oralité.

Dans un autre groupe d'exemples, iam est utilisé pour focaliser des éléments de la phrase. Dans l'exemple suivant, iam est utilisé pour souligner le concept auquel le pronom latin $i d$ se réfère anaphoriquement. Ce concept est introduit dans la proposition antérieure comme quod ex utroque « que à la fois » :

(7) sed quod ex utroque, id iam corpus et quasi qualitatem quandam nominabant (Cic. Ac. 1.24. Kroon \& Risselda 2003, 68)

'mais à la fois, c'est ce qu'ils appelaient corps, ou, si je peux dire, qualité'

Cette phrase est tirée d'une discussion philosophique sur la relation entre la matière informe (materia) et la forme (vis) qui ne peuvent pas exister l'une sans l'autre.

Que iam puisse être considéré comme un marqueur de focus, dans le sens large, peut être prouvé par l'exemple suivant d'une comédie de Térence :

(8) id ita esse vos iam iudicare poteritis (Ter. Eun. 29. Kroon \& Risselda 2003, 72) 
'que c'est le cas sur lequel c'est vous qui déciderez'

Dans la préface de cette comédie, l'auteur défend l'usage de types de caractères parce que des personnages officiels lui avaient reproché d'avoir emprunté ses caractères à d'autres auteurs. À l'aide de iam, il met l'emphase sur vos («vous »), qui désigne le public en opposition aux personnages officiels.

Nous utilisons le terme «marqueurs du focus » dans un sens large, car iam ne marque pas forcément des éléments qui portent l'information «saillante». Il est plus typique que iam attire l'attention sur un élément que le locuteur veut mettre en valeur par rapport au standard ou à une alternative qui est à attendre dans ce cas. L'usage de iam ne se limite pas à la focalisation de certains éléments nominaux, mais peut aussi concerner des relations. Dans l'exemple suivant iam est à côté de l'adverbe corrélatif anaphorique ita («de cette manière ») qui se réfère à la proposition comparative introduite par $u t$ (« comme »). Iam s'utilise, dans cette phrase, pour attirer l'attention sur la comparaison entre deux faits :

(9) Ut igitur paulo ante animum inter Fidenatem Romanamque rem ancipitem gessisti, ita iam corpus passim distrahendum dabis. (Livius 1.28.9. Kroon \& Risselda 2003, 74)

'Ainsi [igitur] comme [ut] ton coeur était partagé entre Fidenae et Rome dans le passé, de la même manière [ita] déjà [iam] ton corps sera déchiré'

Il n'y a pas d'études qui prouvent que la signification temporelle de iam serait la plus fréquente ou prototypique ou que les autres significations se développeraient à partir d'une signification de base. Selon Kroon \& Risselda $(2003,69)$ les données de la langue latine ne donnent pas de points de repère pour supposer un développement diachronique des significations comme marqueur discursif ou marqueur de focus. Elles sont, au contraire, toutes présentes dès les premiers textes.

Sur cette base, nous regarderons le développement des significations de ja/déjà dans Frantext pour vérifier l'hypothèse d'une continuité de celles-ci. Notre hypothèse se répartit en deux parties :

$1^{\circ}$ Les fonctions de connecteur et de marqueur de focus, présentes déjà dans des textes latins à côté de la signification temporelle et de celle du dépassement d'un point de référence sur une échelle, sont continuées dans l'histoire du français.

$2^{\circ}$ Les fonctions de connecteur et de marqueur de focus sont plutôt caractéristiques de la langue parlée et apparaissent, par conséquent, surtout dans des textes conçus oralement. Il s'agit d'une oralité simulée dans des textes écrits qui veulent donner l'impression d'une spontanéité de l'expression.

\section{La polysémie de ja/déjà dans l'histoire du français}

Après avoir analysé les fonctions de iam en latin, nous les comparerons avec celles de ja et déjà en français. Dans cette analyse, nous ne partons pas de l'hypothèse que la continuation de l'étymon garantit la continuité des significations, mais nous ne supposons pas non plus que des significations diverses se soient développées à partir d'une signification de base. La pragmaticalisation parallèle serait prouvée si nous pouvions trouver les mêmes significations dans différentes étapes de l'histoire du français. Des travaux comme Franckel 1989, Garrido 1992, Auwera 1993, 1998, Mosegaard Hansen 2003, Mosegaard Hansen \& Strudsholm 2008 sont orientés vers la langue actuelle, mais, sur une base panchronique, ils prennent aussi en compte des développements dans des époques plus anciennes.

Si nous partons d'une polyfonctionnalité de la particule latine iam et si nous utilisons les fonctions déduites de celle-ci comme critère de comparaison, cette méthode et la correspondance des significations actuelles ne doivent pas cacher la non-identité des emplois dans les époques plus anciennes du français avec les significations de iam et les significations modernes. La différenciation des significations de la particule paraît être un phénomène panchronique qui est basé sur des processus cognitifs, mais qui doit s'établir dans chaque langue. Dans les langues romanes, ce processus s'étend sur plusieurs siècles et il commence à différents moments. Il ne dépend pas des influences d'une langue sur une autre et il a mené, à quelques détails près, au même résultat.

Depuis l'analyse de Martin (1980), la signification de base de déjà est considérée comme aspectuelle. Cette signification grammaticale apparaît dans deux emplois : 
$1^{\circ}$ L'emploi phasique qui exprime la précocité de survenance d'un évènement qui était attendu pour plus tard. On peut voir cette acception dans l'exemple suivant :

(10) Tiens ! Il est déjà six heures et demie ; tu me paies à dîner ? (TLF, 1929, M. Arland, L'Ordre)

Cet emploi absolu de l'adverbe déjà remonte au XIII ${ }^{\mathrm{e}}$ siècle (Välikangas 1985 : 78, Buchi 2007 : 355) :

(11) $[\ldots]$ ge, [...], ai vendu et livré e doné [...] E desja m'en sui desvestuz e dessaziz, e en ai vestu e sazi [...] (1260, charte rédigée à La Rochelle)

$2^{\circ}$ L'emploi itératif de l'adverbe aspectuel déjà consiste à marquer le passé d'expérience (Buchi 2007 : 355). Dans cette acception, l'adverbe apparaît exclusivement avec des verbes composés et il marque qu'à un moment du passé, le procès exprimé dans l'énoncé a eu lieu au moins une fois

(12) Knock. - Vous aviez déjà consulté le docteur Parpalaid ? La dame. — Non, jamais. (TLF, 1923, J. Romains, Knock ou le triomphe de la médecine,)

Cet emploi itératif est attesté la première fois en 1330 (Buchi 2007 : 355-356) :

(13) Comme nostre amé frere Jehan de Saint Laurens, religieux, ceinnier de nostre dite eglise, nous eust des ja piessa ["il y a longtemps"] supplié que nous voulssissions lui ottroier une place (...), avons ottroié et ottroions par ces presentes au dit suppliant sa requeste (1330, Chartes de l'abbaye de Saint-Magloire, BTMF)

\subsection{La contraction des/dès + ja/jà}

L'adverbe ja/jà s'utilisait en ancien et en moyen français, avec les significations 'de facon précoce, déjà maintenant' (14), 'maintenant, en ce moment' (15), 'dans un moment du passé' (16), 'très bientôt, immédiatement, aussitôt' (17), 'toujours' (dans le présent ou le futur) (18), 'jamais' (dans des contextes négatifs, mais sans ne) (19). ${ }^{2}$

(14) Il ne pout mie returner : La nefs est ja en halte mer! (DMF, Marie Guigemar 192)

(15) Et qe totes maners de noire monoie, qe courent ja communalment en noz roialme ou poer, soient tote outrement ostez (DMF, Stats i 273)

(16) Si cum li aunceisor furent ja costumier (DMF, Horn 4324)

(17) El demaunde sovent: 'Dan Herland, quant vendra?' E eles li responent: 'Ja, quant servi avra' (DMF, Horn 530)

(18) la mole desuz [...] si [ne] puet ele esbrissir, e ja soit de l'autre dessevré, si [ne] la put hom assembler (DMF, A-N Med i 59.xlvii)

(19) la rose qe ja enflestrith (DMF, BIBB ANTS 1042)

Avec une négation, il signifiait 'jamais' et, dans des phrases négatives ou optatives, il était utilisé avec la signification de certes. L'emploi de la forme ja/jà continue jusqu'au XVI siècle quand il est substitué par desjà/déjà qui entre en concurrence avec ja/jà au XIII siècle (cf. Martin 1980). Le Trésor de la langue française évoque une trace de cet emploi dans ce pastiche de Flaubert imitant le langage de Rabelais :

(20) Or donc, puisque n'avons jà bronché (estant ferrés à glace, ie suppose) ni jà courbé nostre eschine sous le linteau d'aulcune boutique, ecclise, confrayrie, servition quelconque, guardons (ce est mon souhait de nouvel an pour tous deux) ceste sempiternelle superbe amour de beaulté (TLF, Flaubert, Correspondance, 1852 : 75).

L'adverbe déjà est le résultat d'une contraction et d'un changement de sens de la locution des ja qui était utilisée avec la signification « dès à présent » (ca. 1275 Jean de Meun, Rose, éd. F. Lecoy, v. 19189), et aussi avec la signification « dès ce moment là (du passé) » (1465 Commynes, Mémoires, éd. Calmette, t. 1, p. 22) (TLF, « déjà »). Dans les deux exemples suivants la locution des ja sert à marquer un point avancé dans l'échelle temporelle :

\footnotetext{
${ }^{2}$ Sur le développement de l'emploi de ja cf. Martin 1980.
} 
(21) Comme nostre amé frere Jehan de Saint Laurens, religieux, ceinnier de nostre dite eglise, nous eust des ja piessa supplié que nous voulssissions lui ottroier une place situee es fins du jardin du dit ceinnier (Frantext ${ }^{3}$ 8203, Chartes de l'abbaye de Saint-Magloire, 1330, 740)

'Comme notre frère aimé Jehan de Saint Laurens, moine, personnage officiel de l'église mentionnée, nous avait déjà longtemps demandé que nous lui accordions une place située dans le jardin du personnage officiel mentionné'

Cette signification temporelle-scalaire est partagée par toutes les occurrences de la locution des ja que nous avons trouvées dans Frantext. Le corpus ne présente qu'un seul exemple de cette locution après la première moitié $\mathrm{du} \mathrm{XV}^{\mathrm{e}}$ siècle qui correspond, peut-être, à un usage archaïsant :

(22) [...] quelques uns de nos autheurs, si leur reputation qui volle par toute la France, et que les Alpes, les Pirennées, et l'ocean n'ont pû contenir, ne l'avoit des ja mieux faite que moy. (Q776, Scudéry, L'Apologie du théâtre, 1639, 65)

À partir de la fin du $\mathrm{XV}^{\mathrm{e}}$ siècle, la locution semble être devenue un mot contracté nouveau, déjà, qui apparaît dans plusieurs variantes orthographiques et qui assumera le rôle qu'avait eu iam en latin. Mais avant d'étudier les significations de déjà, il faut regarder l'usage du successeur étymologique immédiat de iam dans des textes plus anciens, la particule ja (jà). Est-ce qu'elle était capable d'exprimer toutes les fonctions de iam et pourquoi a-t-elle disparu en faveur de déjà ?

\subsection{La signification temporelle pure}

La signification temporelle pure de ja est documentée dès les premiers textes :

(23) Pur le soen Deu qu'il ait mercit de mei. Ja einz ne verrat passer cest premer meis Que jel sivrai od mil de mes fedeilz [...] (La Chanson de Roland, Première partie La Mort de Roland, VI, v. 82-83)

'qu'au nom de son Dieu il me fasse merci ; qu'il ne verra point ce premier mois passer que je ne l'aie rejoint avec mille de mes fidèles'

Nous regardons comme purement temporels des usages dans lesquels l'adverbe désigne une période plus ou moins éloignée dans le passé ou le futur. Cette signification n'est pas la plus fréquente dans les textes anciens, mais elle est utilisée avec une certaine régularité.

La particule contractée desjaldéjà est utilisée, depuis la fin du $\mathrm{XV}^{\mathrm{e}}$ siècle avec cette signification :

(24) Mais il y a une chose dont je ne suis pas contant. Car vous aves desja fait l'ommage au roy de Castille des iles Canariennes et vous en distes du tout seigneur; [...] (6206, Béthencourt, Le Canarien, c.1490, 215)

En français moderne, la référence à une échelle temporelle est devenue prototypique. C'est pourquoi déjà, dans sa signification temporelle pure, est souvent utilisé avec une autre expression de temps, comme au printemps dans le cas suivant :

(25) Il reconnut l'anglaise qui, déjà, au printemps, avait accompagné Mme De Battaincourt et sa fille. (K365, Martin du Gard, Les Thibault 1928, 1072)

Comme c'était déjà le cas en latin, des adverbes en fonction purement temporelle peuvent être utilisés avec d'autres expressions temporelles qui indiquent le moment du déroulement ou la longueur de l'évènement :

(26) Soyons juste: depuis déjà longtemps il n'y avait plus grand-chose dedans. (R022, Bazin, L'école des pères, 1991, 340)

Dans de tels usages, si une comparaison avec un point temporel de référence et le marquage de l'évènement comme précoce peuvent jouer un certain rôle, le rapport à un intervalle concret met

\footnotetext{
${ }^{3}$ Tous les exemples suivants sont issus de la Base textuelle Frantext (http://www.frantext.fr/), sauf ceux pour lesquels nous avons noté spécialement leur provenance.
} 
cependant le relatif de la particule temporelle à l'arrière-fond. L'usage d'un nom personnel avec une référence à une époque peut assumer le même rôle désambigüisant :

(27) Voltaire avait déjà pris le parti d'Athalie contre le grand prêtre. (L429 - Mauriac, La Vie de Jean Racine 1928, 194)

\subsection{Référence à une échelle temporelle}

$J a$ s'utilise le plus souvent, dans les textes du XII ${ }^{\mathrm{e}}$ siècle, comme référence à une échelle temporelle où un évènement a dépassé un certain point standard :

(28) En ceste tere ad estet ja VII anz. (La Chanson de Roland, ca. 1125, CXCII)

'Voilà sept ans que l'empereur est en ce pays'

(29) Ne le vous disoie je bien, fait Merlins, qu'elle ne vous averoit ja duree? (R019, La suite du Roman de Merlin, ca. 1235-1240, 49)

'Est-ce que je ne vous ai pas dit, dit Merlin, qu'elle accomplissait sa parole depuis longtemps déjà ?'

La référence à l'échelle temporelle est aussi donnée dans le cas où la particule se réfère à un moment qui coïncide avec le moment de la parole. La particule marque l'accomplissement d'une action ou d'un processus, plus tôt par rapport au temps standard.

Pour la signification temporelle-phasale que nous avons subordonnée à la signification scalaire, plusieurs auteurs ont donné des exemples pour le français et d'autres langues: Traugott \& Waterhouse 1969, Doherty 1973, Muller 1975, König 1977, Hoepelman \& Rohrer 1980, Martin 1978 et 1980, Vet 1980, Välikangas 1982, Löbner, 1989, 1999; Garrido 1992; Vandeweghe 1992; Michaelis 1992, 1996; Auwera 1993, 1998 ; Paillard 1992. En effet, la fréquence de cette signification et la possibilité d'en déduire les autres significations suggèrent l'identification de la signification temporelle-phasale avec le prototype.

Une phrase comme Il dort déjà ? indique qu'un état est atteint ou qu'une action est accomplie. En même temps déjà sert à évaluer la relation à un point de référence sur l'échelle temporelle. Est-ce qu'il dort déjà alors qu'on aurait pu s'attendre, d'après les habitudes de l'individu, à ce qu'il s'endorme plus tard ? Doherty (1973 : 175) et Klein (1992) ont constaté que la particule peut marquer le début d'une action avant le temps topique. Nous entendons par temps topique le moment auquel une relation est établie.

La particule contractée déjà, établit le plus souvent une référence à une échelle temporelle. Déjà indique la terminaison d'une action ou d'un processus avant un moment présupposé comme temps topique. Dans la phrase suivante, ce temps topique correspond au moment de la parole simulée :

(30) [...] non, moi, je vous ai déjà dit souvent que je ne peux pas. (K688, Maurois Climats, 1928, 73)

Tandis que les formes perfectives du verbe renvoient à l'achèvement d'une action, les formes imperfectives permettent aussi une représentation rétrospective. Dans ce cas, on se réfère aussi à un intervalle antérieur sur l'échelle temporelle, mais sans évoquer le début ou la fin de l'action. Par conséquent, celle-ci peut encore durer lors du temps topique :

(31) Enfin, quelques jours avant la date fixée, la presse revint à la charge: "on se battait déjà pour les places..." (K299, Martin du Gard, Devenir, 1928, 71)

\subsection{Qualités scalaires non temporelles}

Comme plusieurs qualités peuvent être conçues d'une manière scalaire, la transmission métaphorique de la particule ja sur certains degrés de ces qualités est probable. Dans l'exemple suivant, elle indique le degré plus haut de beauté :

(32) Ensurquetut si ai jo vostre soer, Sin ai un filz, ja plus bels n'en estoet, Ço est Baldewin, ço dit, ki ert prozdoem (La Chanson de Roland, Première partie La Mort de Roland, vers 312 à 314)

'Sur toutes choses, j'ai pour femme votre sœur. J'ai d'elle un fils, le plus beau qui soit. C'est Baudoin, dit-il, qui sera un preux.' 
Avec Traugott et Dasher (2002 : 3) on pourrait supposer une transposition métonymique de l'échelle temporelle dans la mesure où le mouvement objectif le long de celle-ci implique un mesurage subjectif selon une échelle de valeur - de la beauté en ce cas. Mosegaard Hansen \& Strudsholm (2008: 487) constatent un tel usage pour la particule déjà à la fin $\mathrm{du}_{\mathrm{XIX}}^{\mathrm{e}}$ siècle. Évidemment la non prise en compte de textes d'époques plus anciennes a mené à la constatation d'une grammaticalisation tardive de déjà avec cette fonction. Comme nous l'avons vu, ja continuait cette signification et nous avons même trouvé dans Frantext un exemple avec déjà, datant du XVI siècle :

(33) Par quoi, toute la compagnie se leva et puys nous estantz dehors et moi déjà assez loin, [...] (S359, Le Journal de Guillaume Paradin ou la Vie en Beaujolais au temps de la Renaissance (vers 1510-1589), 1573, 69)

'Par quoi, toute la compagnie se leva et puis, nous étant dehors et moi, déjà assez loin [...].'

Cet usage de déjà repose sur une transposition du concept de temps sur l'espace, bien qu'une certaine notion de temps subsiste. Encore une fois on peut supposer un pont métonymique parce que l'éloignement se produit dans le temps. Il paraît plus judicieux de parler d'une réutilisation successive de la métaphore temporelle qui permet la transposition à d'autres relations scalaires comme celle des distances (déjà assez loin) ou l'aptitude de quelque chose à servir à un certain but (C'est déjà bien! En fait, c'est même super!).

L'exemple suivant, utilisé par Buchi (2007 : 356) pour l'emploi scalaire, peut être interprété avec un sens double : le temps est déjà avancé, mais le degré de témérité, lui aussi, a atteint un point très haut. Il s'agit donc d'un contexte-pont entre la signification scalaire temporelle et la signification scalaire nontemporelle :

(34) C'estoit desja beaucoup que d'encourir la haine Des grands Dieux immortels et dela gent Romaine. (TLF, 1604, Montchrestien, Tragédies)

Il y a un autre usage de la particule déjà qui n'apparaît qu'au XIX ${ }^{\mathrm{e}}$ siècle et que Mosegaard Hansen \& Strudsholm $(2008$, 488) décrivent comme marquage de la marginalité d'un représentant prototypique d'une classe, en utilisant l'exemple suivant :

(35) Menton, c'est déjà la France.

Tandis qu'une phrase comme ??Paris, c'est déjà la France serait peu acceptable, l'exemple d'une petite ville dans les Alpes maritimes permet la formulation d'un exemple marginal avec le marqueur déjà. Un rang plus haut ou plus bas sur l'échelle de la marginalité peut être mis en relation avec l'échelle temporelle. Un degré très bas des propriétés prototypiques d'une classe correspond à une position basse sur l'échelle subjective.

\subsection{Signification polaire}

Nous avons mentionné, pour le iam latin, la signification polaire qu'on pourrait interpréter comme dépassement du possible sur une échelle. La continuité de cette signification peut être observée dans la particule $j a$ des textes en ancien français :

(36) Si l'orrat Carles, ki est as porz passant. Je vos plevis, ja returnerunt Franc. (La Chanson de Roland, Première partie La Mort de Roland (vers 1071 à 1072)

'Charles l'entendra, qui est au passage des ports. Je vous le jure, les Français reviendront déjà.'

(37) Mais c'est folie de beer a l'avoir, car ja sans faille ne l'averés se par moi n'est. (R019, La suite $d u$ Roman de Merlin, ca 1235-1240, 51)

'Mais c'est une folie d'aspirer à l'avoir puisque déjà incontestablement tu ne l'accompliras que par moi.'

Quant à la particule contractée déjà, elle se présente, parfois mais rarement, comme indication d'une action présupposée et non actualisée. Nous avons trouvé une signification de ce type en français classique :

(38) Déjà croyant l'imposture, 
ce pere inconsideré

sur la moindre conjecture

tenoit le crime averé. (Q626,Tristan l’Hermite, Les Vers hérö̈ques, 1648, 142)

Cette particule s'utilise surtout pour introduire une opposition sémantique et elle souligne la signification polaire de deux expressions contradictoires dans un contexte. Dans l'exemple de Corneille, la propriété 'presque échappé' est introduite par déjà et ainsi son opposition à 'je rentre dans ma chaîne' est soulignée :

(39) Que pour ton amitié je vais souffrir de peine !

Déjà presque échappé je rentre dans ma chaîne,

Il faut encore un coup m'exposant à ses yeux,

Reprendre de l'amour afin d'en donner mieux. (R951, Corneille, La Place Royale ou L'Amoureux extravagant, 1637, 501)

\subsection{Focalisation}

Le point de départ de l'usage focalisant de la particule était l'usage, courant jusqu'à nos jours, avec des expressions de temps qui sont soulignées par la particule et évaluées par rapport à un temps standard :

(40) Je l'attends déjà depuis deux heures.

On peut trouver quelques occurrences de $j a$ avec marquage du focus dans les textes anciens :

(41) Puis que il sunt as chevals e as armes, $J a$ pur murir n'eschiverunt bataille. (La Chanson de Roland, Première partie La Mort de Roland, vers 1095 à 1096)

'Une fois à cheval et en armes, déjà pour mourir ils n'esquiveront une bataille.'

(42) $\mathrm{Ja}$ Diex nele voelle que tu dies voir de ceste chose. (R019, La suite du Roman de Merlin, ca. 1235-1240, 86)

'Que Dieux ne veuille déjà que tu fasses voir cette chose.'

Les deux derniers exemples sont issus du discours direct de personnages, ce qui souligne leur conception orale.

Nous avons pu trouver des occurrences de la particule contractée desja/déjà au $\mathrm{XV}^{\mathrm{e}}$ siècle :

(43) Semblablement peulx avoir oy par le raport des vielz la tempeste que fist nagueres en Castille Pierre soy disant roy; pour laquelle aggraver il appella a son ayde les payens d'Auffrique et lez gens d'Engleterre, qui tourmenterent les Espaignez maintes anneez, et a grant occision de peuple et desolation de la terre desja presque inhabitee. (0114, Chartier, Le livre de l'espérance 1429 , 142)

'Semblablement, selon le rapport des vieux, tu peux avoir entendu une tempête que fit naguère le soi-disant roi Pierre en Castille ; pour l'aggraver il appela à son aide des païens d'Afrique et le peuple d'Angleterre qui tourmentèrent les Espagnols pendant beaucoup d'années, par la suite, il y avait un grand massacre du peuple une grande désolation du pays déjà presque inhabité.'

(44) Ilz y sont desja tous portez et les aultres craingnent à y entrer. (5602, Bueil, Le Jouvencel 1461, 245)

'Ils y sont déjà tous portés et les autres craignent d'y entrer.'

Dans tous ces exemples, la focalisation se réalise en même temps que l'expression d'une propriété temporelle. Dans la phrase suivante, déjà focalise le sujet :

(45) Mes menaces déjà font trembler tout le monde. (R946 , Corneille, Clitandre ou l'Innocence délivrée, 1632,150)

La particule déjà n'est pas seulement capable de focaliser des constituants d'une phrase, mais aussi des relations et la prédication entière. Ainsi, dans la phrase suivante, le fait que la préface soit jugée comme trop longue est mis en relief :

(46) Cette préface n'est déjà que trop longue pour une comédie. (S891, Corneille, La Veuve : Au lecteur, 1634, 378) 
En espagnol, la particule ya peut être utilisée pour renforcer l'impératif (;Cállate ya!), ce qu'on pourrait expliquer comme un élargissement de la signification temporelle : le locuteur exige le changement rapide d'un état (Garrido 1992). Mosegaard Hansen \& Strudsholm (2008: 497) mentionnent un cas du même comportement pour la particule française déjà, et ils l'expliquent par le contact avec l'espagnol dans un texte de Flaubert :

(47) [...] tu l'as voulu, disait-il, tu l'as voulu ! Tu as attaché ta vie à la mienne. Vois déjà ! (M728, Flaubert, La Première éducation sentimentale, 1845, 177)

Nous avons trouvé deux autres exemples dans Frantext, ce qui nous incite à parler d'une signification potentielle en français, mais qui est rarement utilisée :

(48) Vois déjà sa servante, elle revient. (R315, Corneille, La Suite du Menteur, 1682, 324) Et vois déjà ce qui s'est introduit par cette fissure adventice ! (R 252, Claudel, Commentaires et exégèses, 1948, 204)

Dans un autre emploi, focalisant aussi, mais en même temps conjonctif et marquant l'ordination, déjà apparaît soit en détachement en début de proposition, soit suivi de que (Mosegaard Hansen 2002 : 41-45 : Buchi 2007 : 356) :

(49) J'ai bien aimé ce film : déjà, c'est original, et puis il y a de très belles photos (Mosegaard Hansen $2002: 42$ )

(50) Madame Des Pereires, fort nerveuse, essayait de remettre un peu d'ordre... que ça ait pas l'air trop étable... déjà que c'était normalement une terrible pétaudière, alors depuis cette cohue, y avait plus un sifflet d'espace! (S984, L.-F. Céline, Mort à crédit, 1936)

Dans cet emploi, la proposition contenant déjà appelle obligatoirement une suite et, d'autre part, un énoncé comportant déjà peut mettre sur le même plan des entités dissemblables (Mosegaard Hansen 2002 : 42 : Buchi 2007 : 357) :

(51) J'aime bien cet appart : déjà, il est super-bien situé, puis il est grand, et enfin il n'est pas cher. (Mosegaard Hansen 2002: 42

Buchi (2007 : 357) a pu relever une première occurrence de cette acception qui remonte au début du $\mathrm{XX}^{\mathrm{e}}$ siècle :

(52) Déjà, en France, je ne passe pas trop facilement, mais là-bas, si vous saviez ! (R608, L. Bloy, Exégèse des lieux communs, 1902)

\subsection{Emploi interactionnel}

Mosegaard Hansen (2002 : 46) décrit un usage interactionnel (interactional usage) dans lequel l'emploi phasique s'élargit au niveau des actes de paroles. Dans l'exemple suivant, déjà marque la demande de rappel d'information et il constate que l'information appelée était connue, mais qu'elle est momentanément oubliée :

(53) Comment c'est le nom de ce pays, déjà ? Bezoncourt ? Bezancourt ? (TLF, J. Giono, Le grand troupeau, 1931)

Selon Välikangas (1985 : 20) et Buchi (2007 : 257), la première attestation absolue de ce tour remonte à la seconde moitié du XVII ${ }^{\mathrm{e}}$ siècle, mais on ne connaît pas d'autre témoignage de l'acception interactionnelle avant la fin du XVIII ${ }^{\mathrm{e}}$ siècle. Cela ne veut pas dire que déjà ne s'employait pas de façon interactionnelle au XVII ${ }^{\mathrm{e}}$ et au XVIII ${ }^{\mathrm{e}}$ siècles, mais qu'il y a peu de textes écrits qui reflètent cet usage oral :

(54) Et comment s'appelle-il ? Ouy, comment s'appelle-il desja ? [équivalent allemand : Und wie heisset er ? Ja/ wie heisst er doch schon ?] (1662/1664, N. Duez, Le vray et parfait Guidon de la langue françoise, Välikangas $1985: 20)$

(55) Comment s'appelle-t-il déjà ? (1790, P. Merle d'Aubigné [Genève, Neuchâtel], Éléments de grammaire françoise, selon Buchi 2007 : 7)

Välikangas suggère que cet emploi interactionnel est probablement dû à un calque de l'allemand : 


\begin{abstract}
J'en arrive à la conclusion que schon a pu se trouver bien plus facilement que déjà une fonction pragmatique nouvelle. Selon toute vraisemblance c'est schon qui a fait naître la locution Wie heisst er schon ? ; ensuite le français, grâce peut-être au rôle syntaxique plus important de déjà, a de son côté généralisé l'emploi d'une locution qui restait en allemand une variante parmi beaucoup d'autres. (Välikangas $1985: 25$ )
\end{abstract}

On peut constater un autre emploi qui relève de l'interaction et qui est né de l'influence de l'allemand. Sur cet emploi, il y a un témoignage métalinguistique de Mauvillon de 1747 (cf. Buchi 2007 : 259) :

\begin{abstract}
Les Allemands emploient dans leur Langue, le mot qui signifie déjà, avec le temps futur, \& il n'y a presque personne parmi eux qui n'en use de-même en parlant François ; c'est une de ces fautes où ils tombent le plus imperceptiblement. Par exemple ils disent : Je ferai déjà en sorte que cela n'arrive pas. Pour parler François, il faudrait dire, Je ferai bien en sorte que cela n'arrive pas. Je ferai bien cela moimême, \& non pas, Je ferai déjà cela moi-même. Je saurai bien le forcer à cela, \& non pas, je saurai déjà le forcer. Je trouverai bien les moyens, \& non pas, je trouverai déjà, \& ainsi du reste. (Mauvillon 1747 : 113-114)
\end{abstract}

Avec cet emploi de déjà, en général en cooccurrence avec un verbe au futur, le locuteur exprime sa conviction qu'un évènement se réalisera.

\title{
4 Continuité du potentiel sémantique et pragmaticalisation parallèle
}

La conformité entre le latin et le français dans la polyfonctionnalité des particules temporelles pourrait être généralisée par une comparaison avec d'autres langues romanes (Haßler 2011, 2013). Elle s'explique par la continuité du potentiel cognitif qui repose sur des relations métonymiques. Ce potentiel sémantique est actualisé, dans les différentes langues, à différentes époques et avec des intensités inégales.

La pragmaticalisation de la particule temporelle iam et ja/jà qui a mené à des fonctions parallèles en latin et en français, se répète en français à partir de la seconde moitié $\mathrm{du} \mathrm{XV}^{\mathrm{e}}$ siècle, pour la particule desja/déjà qui avait marqué d'abord un point sur l'échelle temporelle, mais qui a acquis toutes les autres significations au cours du processus de pragmaticalisation. Cette pragmaticalisation est parallèle en trois sens. Premièrement le français, comme les autres langues romanes, a acquis toutes les significations de la particule latine iam, malgré un certain décalage dans le temps et des variations dans la fréquence. On pourrait appeler ce parallélisme de la pragmaticalisation parallélisme interlinguistique. Deuxièmement la particule contractée déjà a acquis, dès la deuxième partie du $\mathrm{XV}^{\mathrm{e}}$ siècle, toutes les significations qu'avaient la particule iam et la particule ja. Pour désigner ce processus, il semble justifié de parler d'un parallélisme interchronologique. Troisièmement, l'usage de la particule discursive ne contredit pas l'usage comme adverbe temporel. La signification discursive est présente dès les textes latins, ainsi, il est difficile de dire qu'elle se soit développée postérieurement à la signification temporelle $(\mathrm{A} \rightarrow \mathrm{B})$, ni que les différentes significations dépendent du contexte (C[contexte 1] - D[contexte 2]). La pragmaticalisation parallèle consiste, par contre, en l'usage d'un adverbe dans sa signification temporelle et, parallèlement, en l'usage de la même forme comme marqueur discursif $(\mathrm{E}-\mathrm{F})$, tous deux apparaissant de manière autonome dans la langue.

\section{Références bibliographiques}

Auwera, J. van der (1993). Already and still: beyond duality. Linguistics and Philosophy 16, 613-653.

Auwera, J. van der (1998). Phasal adverbials in the languages of Europe. Adverbial Constructions in the Languages of Europe, éd. par J. van der Auwera et D. P. Ó. Baoill. Berlin / New York: Mouton de Gruyter, 25-145.

Buchi, E. (2007). Approche diachronique de la (poly)pragmaticalisation de français déjà ('Quand le grammème est-il devenu pragmatème, déjà ?'). Actes du XXIVe Congrès International de Linguistique et de Philologie Romanes (Aberystwyth 2004), éd. par D. Trotter. Tübingen: Niemeyer, 3, 251-264.

Combettes, B., Schnedecker, C. et Theissen, A. (éd.) (2003) Ordre et distinction dans la langue et le discours, Actes du Colloque international de Metz (18, 19, 20 mars 1999). Paris : Honoré Champion éditeur. 
DMF = Dictionnaire du moyen français. http://www.atilf.fr/dmf/.

Doherty, M. (1973). Noch and schon and their presuppositions. Generative Grammar in Europe, éd. par F. Kiefer, Ferenc et Ruwet, Nicolas. Dordrecht: Reidel.

Dostie, G. (2004). Pragmaticalisation et marqueurs discursifs. Analyse sémantique et traitement lexicographique. Bruxelles : De Boeck-Duculot.

FEW: Französisches etymologisches Wörterbuch: eine Darstellung des galloromanischen Sprachschatzes, éd. par W. v. Wartburg, K. Baldinger, E. Buchi. https://apps.atilf.fr/lecteurFEW/index.php/site/index.

Fløttum, K. (2003). À propos de 'quant à' et 'en ce qui concerne. Ordre et distinction dans la langue et le discours, Actes du Colloque international de Metz (18, 19, 20 mars 1999), éd. par B. Combettes, C Schnedecker et A. Theissen. Paris: Honoré Champion éditeur, 185-202.

Franckel, J.-J. (1989). Étude de quelques marqueurs aspectuels du français. Genève : Droz.

Frantext = Base textuelle Frantext (http://www.frantext.fr/)

Garrido, J. (1992). Expectations in Spanish and German adverbs of change. Folia Linguistica, 26, 357-402.

Haßler, G. (2011). Grammatikalisierung oder Lexikalisierung? Zur Entwicklung von Topik- und Fokusmarkern in romanischen Sprachen. Sprachkontakte, Sprachvariation und Sprachwandel. Festschrift für Thomas Stehl zum 60. Geburtstag, hrsg. v. C. Schlaak et L. Busse. Tübingen: Narr Verlag, 49-68.

Haßler, G. (2013). Von lat. iam zu polfunktionalen pragmatischen Partikeln in romanischen Sprachen. Variatio verborum: Strukturen, Innovationen und Entwicklungen im Wortschatz romanischer Sprachen. Festschrift für Bruno Staib zum 65. Geburtstag, hrsg. v. B. Hillen, S. Jansen et A. Klump. Stuttgart: ibidem-Verlag, 157-175.

Hoepelman, J. et Rohrer, C. (1980). Déjà et encore et les temps du passé du français. La notion d'aspect, éd. par J. David et Martin, R. Metz, Centre d'analyse syntaxique, Université de Metz, 119-143.

Klein, W. (1992). The present perfect puzzle. Language 68, 525-552.

Koch, P.et Oesterreicher, W. (1990). Gesprochene Sprache in der Romania: Französisch, Italienisch, Spanisch. Tübingen: Niemeyer.

König, E (1977). Temporal and non-temporal uses of noch and schon in German. Linguistics and Philosophy 1, 173198.

Kroon, C. et Risselda, R. (2003). Phasality, Polarity, Focality: A Feature Analysis of the Latin Particle iam. Particles [= Belgian Journal of Linguistics 16], éd. par T van der Wouden, A. Foolen et P. van de Craen. Amsterdam: John Benjamins Publishing Company, 65-78.

Löbner, S. (1989). German schon - erst - noch: an integrated analysis. Linguistics and Philosophy 12, 167-212.

Löbner, S. (1999). Why noch and schon are still duals: a reply to van der Auwera. Linguistics and Philosophy 22, 45107.

Marcello-Nizia, C. (2006). Grammaticalisation et changement linguistique. Bruxelles : De Boeck.

Martin, R. (1978). Déjà et encore : de la présupposition à l'aspect. La notion d'aspect, éd. par J. David et R. Martin. Metz: Centre d'analyse syntaxique, Université de Metz, 167-180.

Martin, R. (1980). Sur le destin de jà. Opérateurs et constructions syntaxiques. Évolution des marques et des distributions du XVe au XXe siècle. Actes du colloque international organisé à l'école Normale Supérieure les 11 et 12 décembre 1992 par la GEHLF, éd. par le Groupe d'étude en histoire de la langue française. Paris : Presses de l'école Normale Supérieure, 7-54.

Mauvillon, E. (1747). Remarques sur les germanismes. Ouvrage utile aux Allemands, aux François et aux Hollandois, \& c. Amsterdam : Pierre Mortier.

Menge, H. (2000 [1873]). Lehrbuch der lateinischen Syntax und Semantik, völlig neu bearbeitet von Th. Burkard und M. Schauer, wissenschaftliche Beratung F. Maier. Darmstadt: Wissenschaftliche Buchgesellschaft.

Michaelis, L. A. (1992). Aspect and the semantics-pragmatics interface: the case of 'already'. Lingua 87, 321-339.

Michaelis, L. A. (1996). On the use and meaning of already. Linguistics and Philosophy 19, 477-502. 
Mosegaard Hansen, M.-B. (1998). The function of discourse particles. A study with special reference to spoken standard french. Amsterdam, Philadelphia : Benjamins

Mosegaard Hansen, M.-B. (2002). From Aspectuality to Discourse Marking : the Case of French déjà and encore. Belgian Journal of Linguistics 16, 23-51.

Mosegaard Hansen, M.-B. (2003). From aspectuality to discourse marking: the case of French déjà and encore. Particles [= Belgian Journal of Linguistics 16], éd. par T. van der Wouden, A. Foolen et P. van de Craen. Amsterdam: John Benjamins Publishing Company, 23-51.

Mosegaard Hansen, M.-B. et E. Strudsholm (2008). The semantics of particles: advantages of contrastive and panchronic approach: a study of the polysemy of French déjà and Italian già. Linguistics 46, 471-505.

Muller, C. (1975). Remarques syntactico-sémantiques sur certains adverbes de temps. Le français moderne 43, 12-38.

Nørgård-Sørensen, J., L. Heltoft et L. Schøsler (2011). Connecting Grammaticalisation. Amsterdam/Philadelphia: John Benjamins Publishing Company.

Paillard, Denis (1992). Déjà et la construction de l'énoncé. L'Information grammaticale 55, 33-37.

Pusch, C. D. (2007). Les corpus de linguistique romane en pays germanophones. Bilan et perspectives. Revue française de linguistique appliquée 12, 111-124.

TLF = Trésor de la Langue Française. http://atilf.atilf.fr/.

Traugott, E. C. et Dasher, R. B. (2002). Regularity in semantic change. Cambridge: Cambridge University Press.

Traugott, E. C. et Waterhouse, J. (1969). Already and yet: a suppletive set of aspect-markers?. Journal of Linguistics 5, 287-304

Välikangas, O. (1982). La notion de déjà et les mots qui servent à la rendre dans quelques langues européennes. Neuphilologische Mitteilungen 83, 371-404.

Välikangas, O. (1985). Paradigmes logiques et contacts de langues : à propos de déjà, encore et schon, noch. ACILPR XVII, vol. 7, 17-26.

Vandeweghe, W. (1992). Perspectivische evaluatie in het Nederlands : De partikels van de AL/NOG/PAS-groep. Ghent: Koninklijke Academie voor Nederlandse Taal- en Letterkunde.

Vet, C. (1980). Temps, aspect et adverbes de temps en français contemporain. Geneva: Droz.

Waltereit, R. (1998). Metonymie und Grammatik. Kontiguitätsphänomene in der französischen Satzsemantik. Tübingen, Niemeyer.

Waltereit, R. (2006). Comparer la polysémie des marqueurs discursif. Les marqueurs discursifs dans les langues romanes. Approches théoriques et méthodologiques, éd. par M. Drescher et B. Frank-Job. Frankfurt : Peter Lang, 141-151.

Waltereit, R. (2012). On the origins of grammaticalization and other types of language change in discourse strategies. Grammaticalization and Language Change: New reflections, éd. par K. Davidse, T. Breban, T., L. Brems et T. Mortelmans. Amsterdam, Philadelphia: John Benjamins Publishing Company, 51-72.

Wouden, T. van der, A. Foolen et P. van de Craen (2003). Particles [= Belgian Journal of Linguistics 16, 2002]. Amsterdam: John Benjamins Publishing Company. 